\title{
Conductance and Capacity of Plain Lipid Membranes under Conditions of Variable Gravity
}

\author{
Michaela Sieber, Stefan Kaltenbach, Wolfgang Hanke, Florian P. M. Kohn* \\ Department Membrane Physiology, Institute of Physiology, University of Hohenheim, Stuttgart, Germany \\ Email: *florian.p.m.kohn@uni-hohenheim.de
}

Received 11 April 2016; accepted 26 June 2016; published 30 June 2016

Copyright (C) 2016 by authors and Scientific Research Publishing Inc.

This work is licensed under the Creative Commons Attribution International License (CC BY). http://creativecommons.org/licenses/by/4.0/

(c) (i) Open Access

\begin{abstract}
Biological cell membranes are complex structures containing mainly lipids and proteins. Functional aspects of such membranes are usually attributed to membrane integral proteins. However, it is well established that parameters of the lipid matrix are modifying the function of proteins. Additionally, electrical capacity and conductance of the plain lipid matrix of membranes are contributing directly to cellular functions as there is, for example, the propagation of action potentials. Accordingly the dependence of these parameters on changes of gravity might be important in the field of life sciences under space conditions. In this study consequently we have performed experiments in parabolic flight campaigns utilizing the patch-clamp technology to investigate conductance and capacity of plain lipid vesicle membranes under conditions of changing gravity. Both capacity and conductance were found to be gravity dependent. The changes in capacity could be contributed to changes in membrane geometry. Significant permeability in plain lipid membranes could be only observed at high potentials, where spontaneous current fluctuations occurred. The probability of these fluctuations was gravity dependent.
\end{abstract}

\section{Keywords}

Planar Patch-Clamp Technology, Microgravity, Plain Lipid Membranes, Bilayer, Conductance, Capacity

\section{Introduction}

Biological systems are known to interact with gravity on different levels of organization, from the whole organ-

\footnotetext{
${ }^{*}$ Corresponding author.
}

How to cite this paper: Sieber, M., Kaltenbach, S., Hanke, W. and Kohn, F.P.M. (2016) Conductance and Capacity of Plain Lipid Membranes under Conditions of Variable Gravity. J. Biomedical Science and Engineering, 9, 361-366. 
isms [1] [2] to cells [3], to membranes [2] and even down to the function of single proteins, especially ionchannels [4]-[6]. Nevertheless, related to the question how membranes and single protein function are affected by gravity there are still a variety of problems existing in interpretation, which concern the basic underlying mechanisms. Membranes are complex systems mainly being composed of lipids and proteins. Usually membrane function is contributed to the proteins which, however, might be affected by the physic-chemical parameters of the lipid matrix. This is exclusively assumed in case of membrane conductance which is stated to be the result of integral membrane proteins involved in ion-transport across membranes [7]. Necessarily, in case physicochemical parameters of membranes themselves are gravity dependent, this seriously would change the cellular response to changes in gravity. As a consequence it should be asked whether plain lipid membranes do interact with gravity. Parameters of such plain lipid membranes being of interest are conductance and capacity.

The conductance of plain lipid membranes is usually low, but at high potentials current fluctuations have been shown to be present [8] [9]. Also, it has been shown that in the temperature range of phase transitions current fluctuations show up even at lower potentials [10]. Both findings indicate that a shift in the phase state of the lipid membrane might result in significant conductance changes, possibly due to dielectric breakdown of the membrane [11] or due to the introduction of phase boundaries.

The capacity of membranes is depending on geometry (area) and on thickness, both parameters are usually only changing on a longer time scale [12], but also on applied potential and phase-state.

Both, membrane conductance and capacity are involved, for example, in the propagation of action potentials [12]-[16] which have already been shown to be gravity dependent in their parameters [17] [18]. Thus, a gravity dependence of membrane conductance and capacity would in example force changes in action potential parameters, and of course a wide range of other membrane related biological functions.

A well-established method to measure membrane conductance and capacity of cellular membranes is the planar patch-clamp technique [19] [20], which has been adapted to parabolic flight conditions in previous studies [2]. It easily can also be used with plain lipid vesicles, in this mode it is equivalent to a bilayer set-up [21] [22]. Thus, in this study a semi-automated patch-clamp set-up is used in parabolic flight missions to detect possible changes in plain lipid bilayer conductance and capacity related to gravity changes.

\section{Materials and Methods}

\subsection{Materials}

All substances and solvents used in the experiments were purchased from Sigma-Aldrich at least of p.a. grade. Distilled water was from a laboratory distillery. Asolectin, a lipid extract from soybean membranes enriched in phosphatidylcholine, was purchased from Sigma-Aldrich.

\subsection{Vesicle Preparation}

For the preparation of the vesicles, lipids $(20 \mathrm{mg} / \mathrm{ml})$ were dissolved in a solvent-mixture (chloroform: methanol 2:1). The fluorescent dye DPH (10 $\mathrm{MM}$, dissolved in Chloroform) was also added. $9 \mathrm{ml}$ of this solution were filled in a flask and the solvent-mixture was removed in a rotary evaporator (400 mbar, $1 \mathrm{~h}$ ) until the lipid was a thin film. To remove the last residue of solvent, the flask was stored in a desiccator for $2 \mathrm{~h}$. Afterwards the lipid-film was rehydrated with $9 \mathrm{ml}$ of buffer (100 mM KCl, $10 \mathrm{mM}$ Tris, $\mathrm{pH}$ 7.4), until the lipid has completely come off the wall. The solution was stored overnight in a fridgeat $4^{\circ} \mathrm{C}$. The rehydrated lipid was sonicated for 30 min and afterwards centrifuged for $10 \mathrm{~min}(2800 \mathrm{~g})$ at room temperature $\left(23^{\circ} \mathrm{C} \pm 1^{\circ} \mathrm{C}\right)$. For a usable vesicle concentration, the supernatant was diluted 1:10 with buffer. All the procedures were done with a minimum of light.

The liposomes were prepared before the flight and $300 \mu \mathrm{l}$ of liposome solution were filled into the wells of the plate. Additionally the reference wells were filled with aqueous solution, and then the plate was tightly sealed by an adhesive plastic foil. For the liposome measurements the plate reader was set to the top-read mode.

\subsection{Patch-Clamp Set-Up}

The experiment is designed on the basis of a Nanion Port-a-Patch [21] set-up, which has been used previously in patch-clamp experiments in parabolic flight missions [2]. This set-up is used to do experiments with plain lipid vesicles, whichis equivalent to bilayer experiments. It was constructed following the rules for experimental 
equipment in parabolic flight campaigns [23].

The set-up consists of two racks, one containing the computer and part of the electronics. The other rack houses the box in which the bilayers experiments are done and the patch-clamp amplifier. Bilayers are made in the set-up from vesicles which are spread on a chip. The vesicles are made from asolectin previous to the flight in the lab on an apparatus designed for this purpose from Nanion (Vesicle Prep Pro) with diameters of about 10 to $20 \mu \mathrm{m}$. The vesicle suspension is applied to the chip, one vesicle is attached to a small hole in the chip by slight low-pressure pulses, the vesicle is stripped of and a planar membrane remains attached to the chip over the hole, allowing classical electrophysiological studies of this plain lipid membrane. In this situation membrane capacity and conductance are continuously monitored at different gravity levels in the voltage clamp mode. In part of the experiments, trains of voltage pulses of constant height were applied to the bilayer to thus record its capacity. The temperature of the experiments was $23^{\circ} \mathrm{C} \pm 1^{\circ} \mathrm{C}$. A photo of the set-up is given in Figure 1.

\subsection{Gravity Levels}

Gravity levels were used as given by the flight protocol of the parabolic flight mission, thus at $1 \mathrm{~g}, \sim 1.8 \mathrm{~g}$, and microgravity with $\sim 9 \times 10^{-3} \mathrm{~g}$ (Novespace, 2009).

\subsection{Data Evaluation}

Current recordings at fixed potentials, with and without voltage pulses applied for capacity measurement, were stored on computer using software from Nanion (Munich, Germany) and Heka (Lambrecht, Germany).

The capacity in traces with voltage pulses applied is reflected by the height of the capacitive current spikes. The height of these pulses was averaged for 20 sec periods each, for $1 \mathrm{~g}$, hypergravity and microgravity phases, and relative changes compared to1g as reference were calculated from these data.

In current traces recorded under voltage clamp conditions without additional voltage pulses, the mean current fluctuation density was integrated after removal of the constant leakage level for intervals of $20 \mathrm{sec}$, again for 1 $\mathrm{g}$, hypergravity and microgravity. The $1 \mathrm{~g}$ value was set as reference, and the results are given relative to this value. Data from two parabolic flight days were used. Out of the given 62 parabolas, 17 have been used at the given potential of $120 \mathrm{mV}$. A paired t-test was applied to test for statistical significance at a significance level of 0.05 .

\section{Results}

In Figure 2 a typical recording of a current fluctuation at a potential of $120 \mathrm{mV}$ is given. Constant voltage pulses are superimposed to the clamping potential in this experiment, resulting in typical capacitive spikes which can be seen in the recording. The height of these spike has be used to obtain a relative measure for membrane capacity under different gravity conditions (see materials and methods section). Also, some current
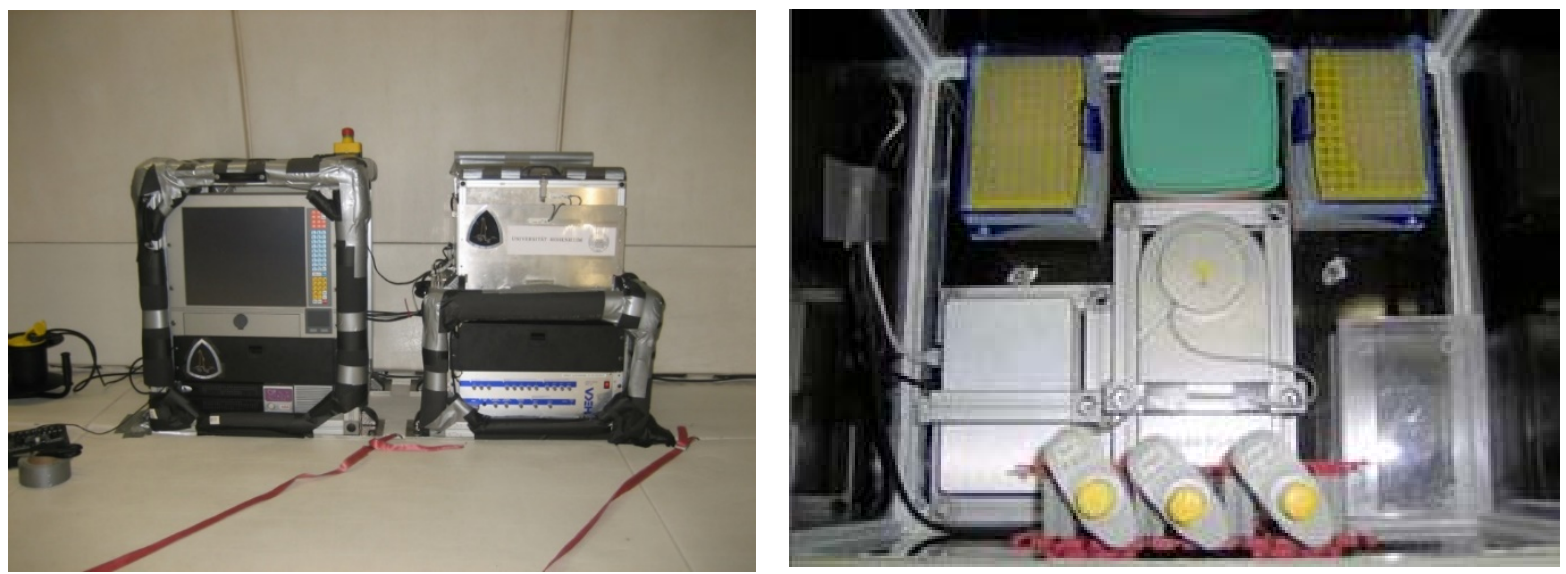

Figure 1. Semi automated patch-clamp (bilayer) set up for parabolic flights based on a Nanion Port-a-Patch system in flight configuration. The patch-clamp set-up itself (right) and the data acquisition system (left) are placed in separate flight racks. 19-inch-rail components have been used. The photo on the right shows the interior of the experimental chamber. 


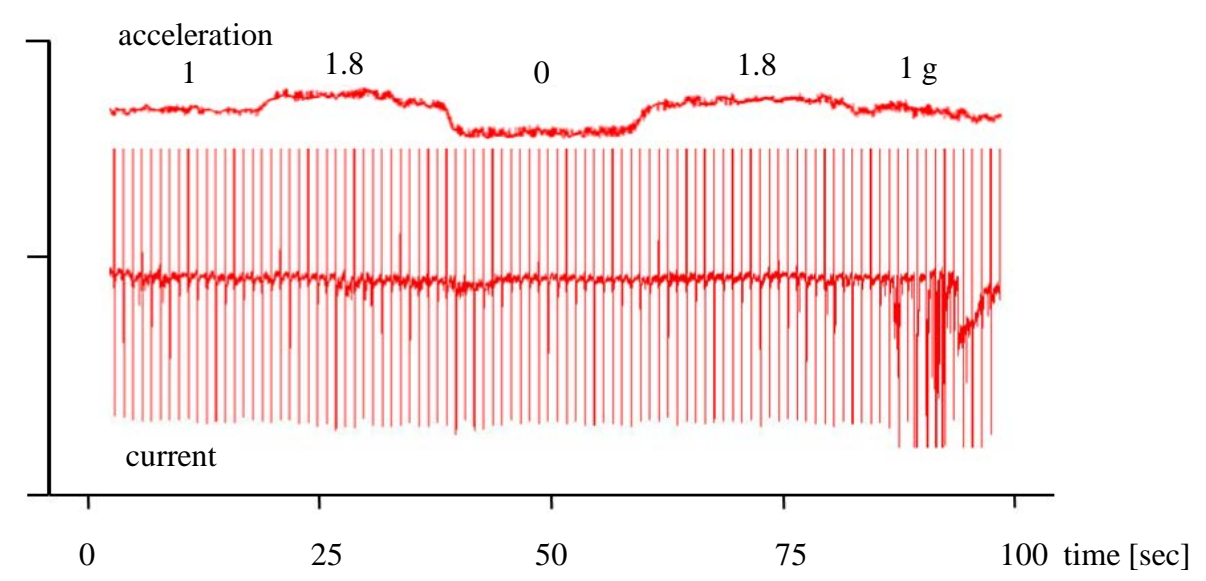

Figure 2. Current recording from an asolectin bilayer at $120 \mathrm{mV}$ with superimposed pulseprotocol for capacity recording. To give a better assignment to the gravity level, a smoothed recording of the actual gravity level of the used parabola is given above the current recording.

fluctuations induced by the relatively high potential can be seen between the spikes, which are less frequent at lower gravity.

From recordings as given in Figure 2, the relative membrane capacity, compared to $1 \mathrm{~g}$, was calculated for hypergravity and for microgravity. The data from a typical parabola are given in the sketch in Figure 3. As it can be seen, changes are not really significant, but when at all, this is due to changes in the geometry (and area) of the bilayer as sketched above the trace.

In Figure 4 in a bar graph, the relative fluctuation density of asolectin bilayers at high membrane potential is given depending on gravity. Data were averaged as stated in the methods section for 17 parabolas. As is obvious from Figure 4, fluctuation density decreases towards lower gravity. The changes are statistically significant (paired t-test at a significance level of 0.05 ).

\section{Discussion}

As is obvious from the results section, both capacity and conductance of plain lipid membranes (bilayers) are depending on the given gravity level. The capacity is slightly bigger than the $1 \mathrm{~g}$ comparison level at both hypergravity and microgravity. Assuming that the bilayer is flat on the glass support of the patch-clamp chip under normal gravity, at lower gravity the bilayer might be slightly curved in upside direction due to the missing force of the water-column. Under hypergravity the water-column has more weight and thus the bilayer might be curved in downwards direction. The effect measured, however, is statistically not significant. Additional effects might be given due to possible changes in the fluidity and thus thickness of the bilayer [24]. Unfortunately, these effects cannot be separated here, however, this possibly can be done in later studies combining electrophysiological with optical methods.

Having a look at the propagation of action potentials along axons, it is found that they slow down under microgravity [17], and speed up at hypergravity. The propagation velocity of action potentials is dependent among other on membrane capacity and on the cross section area of the involved conducting structure. Both are influenced by the gravity level, which should contribute to the explanation of previous results [2] [17].

Membrane conductance of plain lipid bilayers usually is low under physiological conditions. Nevertheless, at high applied potentials current fluctuations can be induced [9] which by some authors are interpreted as lipidchannels [8]. Similar fluctuations, but at more physiological potentials, can be induced in lipid membranes in the range of the phase transition temperature [10] and by addition of hydrophobic substances to the membranes [11] [25]. In all cases these fluctuations are best explained by defects in the membrane structure. In our experiments we induced such current fluctuations by relatively high potentials, $>100 \mathrm{mV}$, and found a decrease of fluctuation density towards lower gravity levels. In previous experiments [24] we have shown that membrane fluidity increases towards lower gravity. This higher fluidity at microgravity might help to faster (better) close potential induced defects in the membrane structure. 


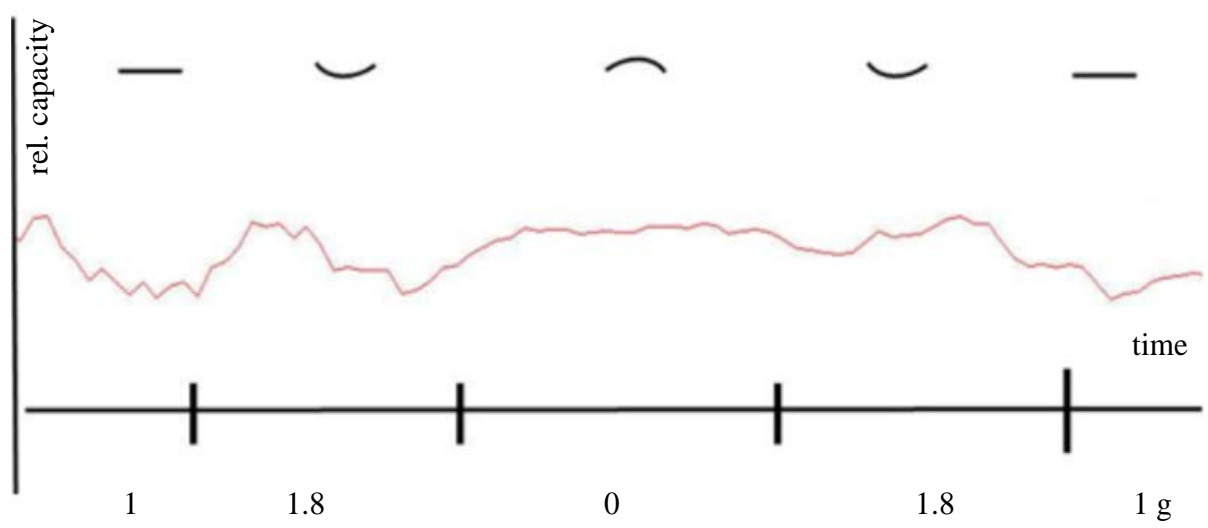

Figure 3. Capacity changes of a bilayer depending on gravity in a parabolic flight. Changes are not significant. Possibly there is a small effect of gravity on bilayer curvature as sketched above the trace.

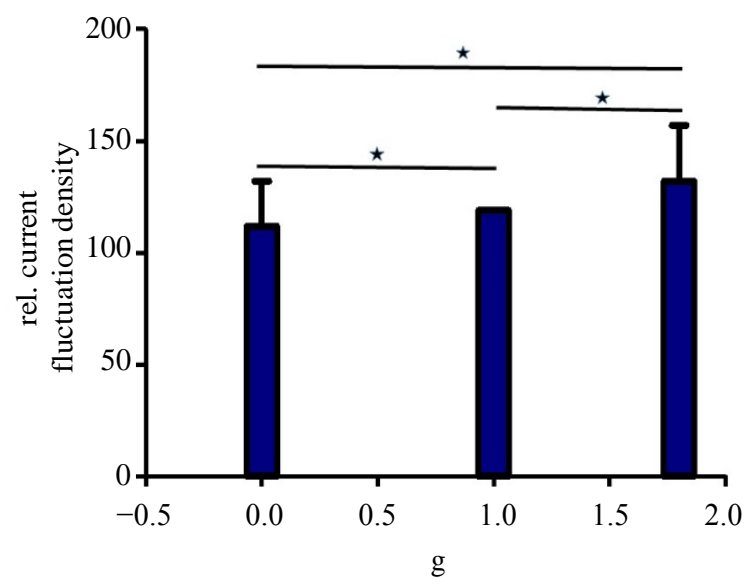

Figure 4. Current fluctuation density at high membrane potential in asolectin bilayers at different gravity levels. Current fluctuation density induced by high potentials is reduced at micro-gravity $(* p \leq 0.05)$.

The physiological relevance of this finding is not so obvious, but in case of hydrophilic substances, for example, a variety of pharmacological relevant drugs are incorporated in the membranes, these also induce structural defects, now at physiological relevant potentials. These defects would be partially restored by microgravity, possibly changing the action of such drugs.

The need of related studies, especially concerning space-pharmacology [26], is an obvious consequence of our findings.

\section{Grants}

This work was supported by Deutsches Zentrum für Luft-und Raumfahrt (DLR) grant 50WB1022.

\section{References}

[1] Hemmersbach, R., Volkmann, D. and Häder, D.P. (1999) Graviorientation in Protists and Plants. Journal of Plant Physiology, 154, 1-15. http://dx.doi.org/10.1016/S0176-1617(99)80311-3

[2] Wiedemann, M., Kohn, F.P.M, Rösner, H. and Hanke, W. (2011) Self-Organization and Pattern-Formation in Neuronal Systems under Conditions of Variable Gravity. Springer Complexity, Springer Publishing Comp., Berlin. http://dx.doi.org/10.1007/978-3-642-14472-1

[3] Häder, D.P., Hemmersbach, R. and Lebert, M. (2005) Gravity and the Behavior of Unicellular Organisms. Cambridge University Press, Cambridge. http://dx.doi.org/10.1017/CBO9780511546211 
[4] Klinke, N., Goldermann, M. and Hanke, W. (2000) The Properties of Alamethicin Incorporated into Planar Lipid Bilayers under the Influence of Microgravity. Acta Astronautica, 47, 771-773. http://dx.doi.org/10.1016/S0094-5765(00)00115-6

[5] Goldermann, M. and Hanke, W. (2001) Ion Channels Are Sensitive to Gravity Changes. Microgravity Science and Technology, 13, 35-38. http://dx.doi.org/10.1007/BF02873330

[6] Wiedemann, M., Rahmann, H. and Hanke, W. (2003) Gravitational Impact on Ion Channels Incorporated into Planar Lipid Bilayers. In: Tien, H.T. and Ottova-Leitmannova, A., Eds., Planar Lipid Bilayers and Their Applications, Elesevier Sciences, Amsterdam, 669-698. http://dx.doi.org/10.1016/S0927-5193(03)80048-7

[7] Weiss, T.F. (1996) Cellular Biophysics: Transport. MIT Press, Cambridge.

[8] Heimburg, T. (2010) Lipid Ion Channels (Review). Biophysical Chemistry, 150, 2-22. http://dx.doi.org/10.1016/j.bpc.2010.02.018

[9] Blicher, A. and Heimburg, T. (2013) Voltage-Gated Lipid Ion Channels. PLoS ONE, 8, e65707. http://dx.doi.org/10.1371/journal.pone.0065707

[10] Gallaher, J., Wodzinska, K., Heimburg, T. and Bier, M. (2010) Ion-Channel-Like Behavior in Lipid Bilayer Membranes at the Melting Transition. Physics Review E, 81, 061925. http://dx.doi.org/10.1103/PhysRevE.81.061925

[11] Wodzinska, K., Blicher, A. and Heimburg, T. (2009) The Thermodynamics of Lipid Ion Channel Formation in the Absence and Presence of Anesthetics. BLM Experiments and Simulations. Soft Matter, 5, 3319-3330. http://dx.doi.org/10.1039/b909877a

[12] Weiss, T.F. (1997) Cellular Biophysics: Electrical Properties. MIT Press, Cambridge.

[13] Hodgkin, A.L. and Huxley, A.F. (1952) The Components of Membrane Conductance in the Giant Axon of Loligo. The Journal of Physiology, 116, 473-496. http://dx.doi.org/10.1113/jphysiol.1952.sp004718

[14] Hodgkin, A.L. and Huxley, A.F. (1952) Currents Carried by Sodium and Potassium Ions through the Membrane of Giant Axon of Loligo. The Journal of Physiology, 116, 449-472. http://dx.doi.org/10.1113/jphysiol.1952.sp004717

[15] Hodgkin, A.L. and Huxley, A.F. (1952) The Dual Effect of Membrane Potential on Sodium Conductance in the Giant Axon of Loligo. The Journal of Physiology, 116, 497-506. http://dx.doi.org/10.1113/jphysiol.1952.sp004719

[16] Hodgkin, A.L. and Huxley, A.F. (1952) A Quantitative Description of Membrane Currents and Its Application to Conduction and Excitation in Nerve. The Journal of Physiology, 117, 500-544. http://dx.doi.org/10.1113/jphysiol.1952.sp004764

[17] Meissner, K. and Hanke, W. (2005) Action Potential Properties Are Gravity Dependent. Microgravity—Science and Technology, 17, 38-43. http://dx.doi.org/10.1007/BF02870977

[18] Rüegg, D.G., Kakebeeke, T.H. and Studer, L.M. (2000) Einfluss der Schwerkraft auf die Fortleitungsgeschwindigkeit von Muskel-Aktionspotentialen. In: Kelle, H. and Sahm, P.R., Eds., Bilanzsymposium Forschung unter Weltraumbedingungen, WPF, Aachen, 752-759.

[19] Hamill, O.P., Neher, E., Sakmann, B., and Sigworth, F.J. (1981) Improved Patch-Clamp Techniques for High-Resolution Current Recordings from Cells and Cell-Free Membrane Patches. Pflügers Archiv, 391, 85-100. http://dx.doi.org/10.1007/BF00656997

[20] Fertig, N., Blick, R.H. and Behrends, J.C. (2002) Whole Cell Patch Clamp Recording Performed on a Planar Glass Chip. Biophysical Journal, 82, 3056-3062. http://dx.doi.org/10.1016/S0006-3495(02)75646-4

[21] Nanion (2014) The World's Smallest Patch Clamp Setup. Providing Biggest Results. http://www.nanion.de

[22] Hanke, W. and Schlue, W.-R. (1993) Planar Lipid Bilayer Experiments: Techniques and Application. Acad. Press, Oxford.

[23] Novespace (2009) A300 Zero-G Rules and Guidelines, RG-2009-2.

[24] Sieber, M., Hanke, W. and Kohn, F.P.M. (2014) Modification of Membrane Fluidity by Gravity. Open Journal of Biophysics, 4, 105-111. http://dx.doi.org/10.4236/ojbiphy.2014.44012

[25] Blicher, A., Wodzinska, K., Fidorra, M., Winterhalter, M. and Heimburg, T. (2009) The Temperature Dependence of Lipid Membrane Permeability, Its Quantized Nature, and the Influence of Anesthetics. Biophysical Journal, 96, 4581-4591. http://dx.doi.org/10.1016/j.bpj.2009.01.062

[26] Wotring, V.E. (2012) Space Pharmacology. Springer Briefs in Space Development, Springer, New York. 


\section{Submit or recommend next manuscript to SCIRP and we will provide best service for you:}

Accepting pre-submission inquiries through Email, Facebook, Linkedin, Twitter, etc A wide selection of journals (inclusive of 9 subjects, more than 200 journals)

Providing a 24-hour high-quality service

User-friendly online submission system

Fair and swift peer-review system

Efficient typesetting and proofreading procedure

Display of the result of downloads and visits, as well as the number of cited articles

Maximum dissemination of your research work

Submit your manuscript at: http://papersubmission.scirp.org/ 